\title{
Intermediate PSA half-life after neoadjuvant hormone therapy predicts reduced risk of castration-resistant prostate cancer development after radical prostatectomy
}

Yong Jin Kang, Won Sik Jang, Jong Kyou Kwon, Cheol Yong Yoon, Joo Yong Lee, Won Sik Ham and Young Deuk Choi ${ }^{*}$ D

\begin{abstract}
Background: The magnitude and rapidity of the tumor response to androgen deprivation is known to predict the durability of the therapy. We have investigated the predictive value of categorizing patients by the half-life of PSA under neoadjuvant androgen deprivation therapy in patients with biochemical recurrence after radical prostatectomy.

Methods: Medical records of 317 patients who received neoadjuvant androgen deprivation therapy before radical prostatectomy and developed biochemical recurrence were analyzed. The patients were categorized into five groups according to PSA half-life. Risk of developing castration resistance was evaluated by Kaplan-Meier analysis and by Cox proportional risk regression analysis.

Results: The median follow-up duration was 50.1 months (IQR 31.8-68.7) and median PSA half-life was 22.1 days (IQR 12.7-38.4). Comparison of survival curves revealed that patients in the intermediate response group showed significantly lower 5-year castration-resistant prostate cancer rate (37.5\%) compared to non-response and ultra-rapid response groups $(63.6 \%, p=0.007 ; 56.1 \%, p=0.031$; respectively). In the multivariate regression model, intermediate response compared to non-response was associated with significantly reduced risk of castration resistance development (hazard ratio $0.397,95 \%$ confidence interval $0.191-0.823, p=0.013$ ) and overall mortality (hazard ratio $0.138,95 \%$ confidence interval $0.033-0.584, p=0.007$ ). When subcategorized by Gleason score, Kaplan-Meier curve revealed that, in the high Gleason score stratum, 5-year castration-resistant prostate cancer rate for intermediate response group (44.0\%) was exceptionally lower than that in non-response group $(66.7 \%, p=0.047)$, while castration resistance increased in other groups.
\end{abstract}

Conclusion: Short PSA half-life as well as no response after androgen deprivation is associated with increased risk of treatment failure compared to intermediate PSA half-life.

Keywords: Prostate, Neoadjuvant androgen deprivation therapy, Prostate-specific antigen

\footnotetext{
*Correspondence: youngd74@yuhs.ac; YOUNGD74@yuhs.ac

Department of Urology, Urological Science Institute, Yonsei University

College of Medicine, 50-1 Yonsei-ro, Seodaemun-gu, Seoul, Republic of Korea
} 


\section{Background}

Androgen deprivation therapy (ADT) has been the preferred therapy for prostate cancer patients with biochemical recurrence (BCR) after definitive local therapy. Despite the initial tumor suppressing effect, the majority of patients develop resistance to medical castration within 2-3 years, rendering the ADT ineffective [1-3]. Modification of the androgen receptor is believed to be the most important cause underlying castration-resistant prostate cancer (CRPC) development, which enables androgen-independent activation of the androgen receptor or alters its sensitivity [1]. Nevertheless, it is known that the androgen axis continues to play an important role in the function and growth of CRPC $[1,4]$.

Regarding the risk of developing CRPC, there has been a notion that the magnitude [5] and rapidity [6] of the tumor response to ADT can reliably predict the durability of the therapy. Some prior studies suggest that a shorter time to nadir or prostate-specific antigen halflife $\left(\mathrm{PSAT}_{1 / 2}\right)$ during ADT is associated with longer CRPC-free survival and cancer-specific survival [7, 8]. However, there have been some recent studies with contradictory results, reporting shorter remission period and poorer survival in rapid response groups undergone ADT $[9,10]$. Because tumor response to ADT may vary [11] and some prostate cancer cells are known to produce little, if any, prostate-specific antigen (PSA) [12], the resultant PSA kinetics can be heterogeneous in nature [3].

Under the assumptions that the risk of developing CRPC can be measured by analyzing the PSA response to hormone therapy and that castration resistance is heterogeneous among the individual tumor cells within a patient, we investigated the value of the $\mathrm{PSAT}_{1 / 2}$ during neoadjuvant ADT as a predictive factor for CRPC in radical prostatectomy patients who developed BCR.

\section{Methods \\ Patient population}

With approval from the Severance Hospital Institutional Review Board (protocol number 4-2016-0506), the clinical information and follow-up data of patients who underwent bilateral nerve-sparing radical prostatectomy in a single center by a single operator (Y.D.C.) between 2002 and 2014 were collected. Informed consent from the participants was waived by the institutional review board. Patients who received neoadjuvant ADT and developed BCR $(n=348)$ were selected. Patients with missing data $(n=18)$ or those who received adjuvant radiotherapy prior to or on the day of BCR diagnosis ( $n$ =13) were excluded from the cohort, leaving 317 patients for analysis. No patients were given cytotoxic chemotherapy before the confirmation of CRPC.

\section{Neoadjuvant therapy and preoperative parameters}

Neoadjuvant hormone therapy in the study cohort consisted of bicalutamide monotherapy (50 mg bid), administered based on treating physician's decision. Regression due to neoadjuvant therapy was noted on most of the specimens, and in 7 (2\%) patients the regression was so extensive that there were no tumor cells detectable despite the positive results on preoperative biopsy. Median duration of neoadjuvant ADT was 53 (interquartile range [IQR] 35-106) days. Initial PSA was measured at the time of screening immediately before the transrectal ultrasound-guided prostate biopsy. Preoperative serum PSA levels were sampled the day before the operation.

$\operatorname{PSAT}_{1 / 2}$ was calculated by the following formula [13]:

$$
\text { PSAT }_{1 / 2}=d t \times \ln 2 \div(\operatorname{lnPSA} 0-\operatorname{lnPSAt})
$$

where $\mathrm{dt}=$ time interval between measurement (days), $\mathrm{PSA}_{0}=$ initial PSA, and $\mathrm{PSA}_{\mathrm{t}}=$ preoperative PSA at time $t$ after the initial measurement. With use of the ultrasensitive PSA essay, an undetectable level of PSA was considered equivalent to $0.01 \mathrm{ng} / \mathrm{mL}$.

\section{Postoperative follow-up}

A patient was considered to have BCR when a postoperative PSA level $0.2 \mathrm{ng} / \mathrm{mL}$ above the nadir was detected after reaching a nadir PSA value of $0.1 \mathrm{ng} / \mathrm{mL}$. Adjuvant ADT was delivered as a luteinizing hormone releasing hormone (LHRH) agonist alone, a combination of LHRH agonist with antiandrogen, or antiandrogen monotherapy, given at the discretion of the treating urologist (Y.D.C.).

CRPC was defined as three consecutive elevated PSA levels after $\mathrm{BCR}$, or detection of metastasis on radiological images. In the absence of three increased PSA measurements, salvage radiotherapy on equivocal findings in radiological images was also counted as a CRPC event. Time to CRPC was determined by subtracting the date of BCR from the date of CRPC event.

\section{Statistical analysis}

The patient cohort was classified into five groups by PSAT $_{1 / 2}$ in 15-day intervals (non-responders [below 0: PSA increased or unchanged], ultra-rapid responders [0 15], rapid responders [15 30], intermediate responders [30 45], and slow responders [over 45]). Comparison of parameters between groups was performed using Fisher's exact test, Mann-Whitney U test, and Kruskal-Wallis test. Survival analysis was performed by plotting Kaplan-Meier curves with a pairwise log-rank test and by constructing multivariate Cox proportional hazard regression models. All statistical analyses were performed using the Statistical Package for Social Sciences v.22.0 for Windows (SPSS; Chicago, Illinois). A p- 
value $<0.05$ on two-tailed test was considered statistically significant in the current study.

\section{Results}

\section{Baseline characteristics}

Baseline cohort characteristics are listed in Table 1. Median follow-up duration was 50.1 months (IQR 31.868.7). Median initial PSA was $23.2 \mathrm{ng} / \mathrm{mL}$ (IQR 11.7-48.9) and median preoperative PSA was $3.39 \mathrm{ng} / \mathrm{mL}$ (IQR 0.877.66). Median PSAT ${ }_{1 / 2}$ was 22.1 days (IQR 12.7-38.4). The number of patients assigned to each PSAT $_{1 / 2}$ group, in order of increasing PSAT $_{1 / 2}$, was $22(6.9 \%), 82$ (25.9\%), $104(32.8 \%), 40(12.6 \%)$, and $69(21.8 \%)$. There were 64 (20.2\%) pathologic T2, 236 (74.4\%) T3, and $17(5.4 \%) \mathrm{T} 4$ patients in the cohort, with no significant difference among groups $(p=0.623)$. There were $14(4.4 \%)$ patients at or below Gleason score 6, 106 (33.4\%) patients at Gleason score 7, and 197 (62.1\%) patients at or above Gleason score 8. There was no significant difference between groups in terms of Gleason score $(p=0.077)$. There was no significant difference in duration of exposure to antiandrogen between groups, except for the slow response group, which had a median exposure of 70 (IQR 36-314) days compared to 35 (IQR 22-46) days in the nonresponding group $(p=0.004)$.

\section{Kaplan-Meier analysis for CRPC-free survival categorized} by $\mathrm{PSAT}_{1 / 2}$ group

Overall, 147 patients (46.4\%) with BCR developed CRPC. Pairwise analysis of Kaplan-Meier curve (Fig. 1a) revealed that in comparison to non-response group, 5-year CRPC rate of the intermediate response group was significantly lower $(37.5 \%$ vs. $63.6 \%$, respectively; $p=0.007)$. Rapid and slow response groups also showed significantly lower rate of CRPC than non-response group $(41.3 \%, 40.6 \% ; p=$ $0.010,0.041$, respectively). CRPC rate in ultra-rapid responding group was similar $(56.1 \% ; p=0.248)$ to nonresponding group, and the difference with intermediate response group was also significant $(p=0.031)$. Differences between rapid or slow response groups and intermediate response group were not significant $(p=0.478$, 0.376 , respectively).

Analysis of overall mortality yielded similar results (Fig. 1b), with rapid and intermediate response groups showing improved 5 -year mortality rate compared to nonresponding group $(7.7 \%, 2.5 \%$ vs. $27.3 \% ; p=0.016,0.008$, respectively). Although CRPC rate was lower in the slow response group, mortality rate difference with nonresponding group was revealed to be not significant $(5.8 \%$; $p=0.077$ ). Except for non-responding group, there was no significant difference in mortality between intermediate response group and ultra-rapid, rapid, and slow response groups ( $p=0.247,0.338,0.419$, respectively).

\section{Multivariate Cox proportional hazards regression analysis} To control the effects from other known prognostic factors, multivariate models were constructed (Table 2). On univariate analysis, rapid, intermediate, slow response groups ( $p=0.011,0.007,0.032$, respectively), Gleason score $>7(p=0.013)$, and pathologic T4 stage $(p=0.034)$

Table 1 Baseline characteristics

\begin{tabular}{|c|c|c|c|c|c|c|}
\hline & & \multicolumn{5}{|l|}{ PSA half-life categories } \\
\hline & & $\begin{array}{l}\text { Non-responder } \\
(n=22)\end{array}$ & $\begin{array}{l}\text { Ultra-rapid responder } \\
(n=82)\end{array}$ & $\begin{array}{l}\text { Rapid responder } \\
(n=104)\end{array}$ & $\begin{array}{l}\text { Intermediate responder } \\
(n=40)\end{array}$ & $\begin{array}{l}\text { Slow responder } \\
(n=69)\end{array}$ \\
\hline & & Median (IQR)/n (\%) & Median (IQR)/n (\%) & Median (IQR)/n (\%) & Median (IQR)/n (\%) & Median (IQR)/n (\%) \\
\hline \multicolumn{2}{|l|}{ Initial PSA (ng/mL) } & $9.6(7.7 \sim 15.2)$ & $33.0(16.5 \sim 61.6)$ & $23.6(12.3 \sim 47.5)$ & $22.8(12.1 \sim 50.5)$ & $16.0(10.2 \sim 40.9)$ \\
\hline \multicolumn{2}{|c|}{$\begin{array}{l}\text { Immediate preoperative PSA } \\
(\mathrm{ng} / \mathrm{mL})\end{array}$} & $14.3(9.6 \sim 21.1)$ & $0.9(0.4 \sim 2.6)$ & $2.5(0.8 \sim 6.3)$ & $4.6(2.1 \sim 9.2)$ & $5.4(3.7 \sim 10.4)$ \\
\hline \multicolumn{2}{|c|}{ ADT exposure duration (days) } & $35.0(22.0 \sim 46.0)$ & $51.5(37.0 \sim 78.0)$ & 63.5(34.0 112.5) & $51.5(36.0 \sim 149.5)$ & $70.0(36.0 \sim 314.0)$ \\
\hline \multicolumn{2}{|l|}{$\mathrm{PSAT}_{1 / 2}$} & $-196.8(-912.9 \sim-47.2)$ & $11.3(9.9 \sim 12.9)$ & $21.6(18.3 \sim 24.0)$ & 33.4(32.4 38.6) & $94.2(54.9 \sim 148.4)$ \\
\hline \multirow[t]{3}{*}{ Pathologic T stage } & $\|$ & $3(13.6)$ & 13(15.9) & $21(20.2)$ & $11(27.5)$ & $16(23.2)$ \\
\hline & III & 17(77.3) & $64(78.0)$ & $77(74.0)$ & $29(72.5)$ & $49(71.0)$ \\
\hline & IV & $2(9.1)$ & $5(6.1)$ & $6(5.8)$ & $0(0)$ & $4(5.8)$ \\
\hline \multirow[t]{3}{*}{ Pathologic Gleason score } & $\leq 6$ & $2(9.1)$ & $2(2.4)$ & $3(2.9)$ & $3(7.5)$ & $4(5.8)$ \\
\hline & 7 & $5(22.7)$ & 19(23.2) & $43(41.3)$ & 12(30.0) & $27(39.1)$ \\
\hline & $\geq 8$ & 15(68.2) & $61(74.4)$ & $58(58.7)$ & $25(62.5)$ & $38(55.1)$ \\
\hline \multirow[t]{2}{*}{ CRPC } & No & $7(31.8)$ & $36(43.9)$ & $61(58.7)$ & $25(62.5)$ & $41(59.4)$ \\
\hline & Yes & 15(68.2) & $46(56.1)$ & $43(41.3)$ & 15(37.5) & $28(40.6)$ \\
\hline \multicolumn{2}{|l|}{ Time to CRPC (months) } & $13.2(6.1 \sim 21.9)$ & $16.1(8.9 \sim 27.8)$ & $18.8(10.0 \sim 41.2)$ & $32.8(12.6 \sim 50.0)$ & $18.0(6.9 \sim 43.5)$ \\
\hline
\end{tabular}

$P S A$ prostate-specific antigen, IQR interquartile range, $A D T$ androgen deprivation therapy, $C R P C$ castration-resistant prostate cancer, $P S A T_{1 / 2}$ prostate-specific antigen half-life 
(a) CRPC-free survival

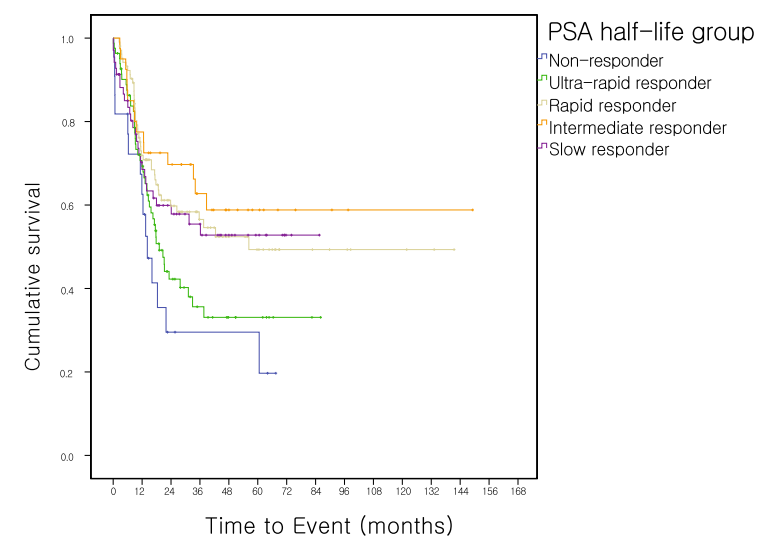

Number at risk:

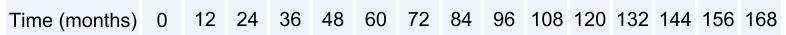

Non-responder $\begin{array}{lllllll}22 & 12 & 4 & 3 & 3 & 2\end{array}$

\begin{tabular}{l|l|l|l|l|l|l|l|l} 
Ultra-rapid & 82 & 46 & 20 & 12 & 7 & 4 & 2 & 1
\end{tabular}

\begin{tabular}{c|l|l|l|l|l|l|l|l|l|l|l|l|l}
$\begin{array}{c}\text { Rapid } \\
\text { responder }\end{array}$ & 104 & 61 & 38 & 26 & 18 & 11 & 7 & 6 & 4 & 3 & 3 & 1
\end{tabular}

responder

\begin{tabular}{|l|l|l|l|l|l|l|l|l|l|l|l|l|l|l} 
Intermediate & 40 & 30 & 22 & 14 & 9 & 6 & 4 & 3 & 2 & 1 & 1 & 1 & 1
\end{tabular}

Sosponder

\begin{tabular}{c|l|l|l|l|l|l|l|l|l|l}
$\begin{array}{c}\text { Slow } \\
\text { responder }\end{array}$ & 69 & 37 & 27 & 19 & 12 & 6 & 2 & 1
\end{tabular}

CRPC: castration-resistant prostate cancer; PSAT12/2: prostate-specific antigen half-life; OS: overall survival

Fig. 1 Kaplan-Meier curve for a CRPC-free survival and $\mathbf{b}$ OS categorized by PSAT (b) os

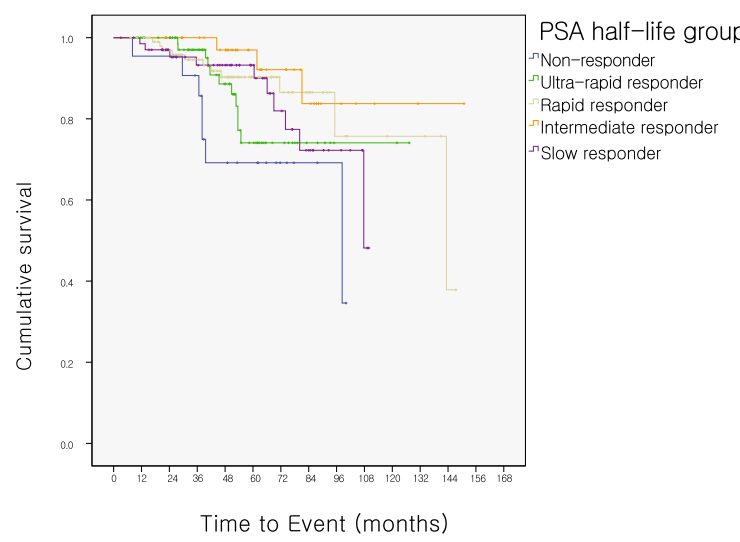

Number at risk

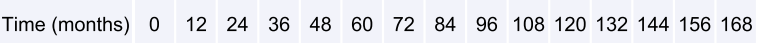

$\begin{array}{lllllllllll}\text { Non-responder } & 22 & 21 & 20 & 17 & 11 & 8 & 4 & 3 & 2\end{array}$

\begin{tabular}{ll|l|l|l|l|l|l|l|l|l|l|} 
Ultra-rapid & 82 & 76 & 65 & 49 & 33 & 17 & 9 & 4 & 2 & 2 & 1
\end{tabular}

\begin{tabular}{ll|l|l|l|l|l|l|l|l|l|l|l|l|l|} 
Rapid & 104 & 97 & 81 & 66 & 46 & 30 & 20 & 12 & 6 & 5 & 4 & 3 & 1
\end{tabular}

responder

\begin{tabular}{ll|l|l|l|l|l|l|l|l|l|l|l|l} 
Slow & 69 & 61 & 52 & 44 & 36 & 26 & 17 & 10 & 5 & 1
\end{tabular} showed significant association with CRPC risk. Age $(p=0.204)$, initial PSA $\geq 20 \mathrm{ng} / \mathrm{mL}(p=0.170)$ and exposure duration $(p=0.169)$ showed no significant association with CRPC. Multivariate Cox regression was performed using variables found significant in the univariate analysis. In the multivariate model, intermediate response to neoadjuvant treatment significantly predicted reduced risk for CRPC (hazard ratio [HR] 0.397, 95\% confidence interval $[\mathrm{CI}] 0.191-0.823, p=0.013$ ) compared to non-responding group. Rapid response was also associated with decreased risk (HR 0.499, 95\% CI $0.271-$ $0.920, p=0.026$ ), but slow response did not show

Table 2 Multivariate Cox regression model for CRPC and overall mortality risk with PSAT $1 / 2$ groups as parameters

\begin{tabular}{|c|c|c|c|c|c|c|c|c|c|}
\hline & & \multicolumn{4}{|l|}{ CRPC } & \multicolumn{3}{|c|}{ Overall mortality } & \multirow[b]{3}{*}{$p$-value } \\
\hline & & \multirow{2}{*}{$\begin{array}{l}\text { Univariate } \\
\text { p-value }\end{array}$} & \multicolumn{3}{|c|}{ Multivariate } & \multirow{2}{*}{$\begin{array}{l}\text { Univariate } \\
\text { p-value }\end{array}$} & \multicolumn{2}{|c|}{ Multivariate } & \\
\hline & & & $\mathrm{HR}$ & $95 \% \mathrm{Cl}$ & $p$-value & & $\mathrm{HR}$ & $95 \% \mathrm{Cl}$ & \\
\hline Age & Continuous & 0.204 & 0.995 & $0.970-1.022$ & 0.734 & 0.243 & 1.046 & $0.990-1.105$ & 0.107 \\
\hline Initial PSA & $\geq 20 \mathrm{ng} / \mathrm{mL}$ & 0.170 & & & & 0.154 & & & \\
\hline Gleason score & $>7$ & $0.013^{*}$ & 1.472 & $1.018-2.128$ & $0.040^{*}$ & $0.006^{*}$ & 3.127 & $1.361-7.187$ & $0.007^{*}$ \\
\hline \multirow[t]{5}{*}{$\mathrm{PSAT}_{1 / 2}$ (days) } & Non-responder (below 0) & (reference) & & & & (reference) & & & \\
\hline & Ultra-rapid responder (0 15) & 0.279 & 0.702 & $0.390-1.263$ & 0.238 & 0.189 & 0.443 & $0.168-1.164$ & 0.099 \\
\hline & Rapid responder (15 30) & $0.011^{*}$ & 0.499 & $0.271-0.920$ & $0.026^{*}$ & $0.018^{*}$ & 0.295 & $0.109-0.797$ & $0.016^{*}$ \\
\hline & Intermediate responder (30 45) & $0.007^{*}$ & 0.397 & $0.191-0.823$ & $0.013^{*}$ & $0.011^{*}$ & 0.138 & $0.033-0.584$ & $0.007^{*}$ \\
\hline & Slow responder (over 45) & $0.032^{*}$ & 0.553 & $0.289-1.057$ & 0.070 & 0.094 & 0.501 & $0.183-1.368$ & 0.177 \\
\hline \multirow[t]{3}{*}{ Pathologic T stage } & Below T2 & (reference) & & & & (reference) & & & \\
\hline & T3 & 0.915 & 0.966 & $0.634-1.471$ & 0.870 & $0.013^{*}$ & 4.987 & $1.187-20.946$ & $0.028^{*}$ \\
\hline & $\mathrm{T} 4$ & $0.034^{*}$ & 1.831 & $0.921-3.642$ & 0.085 & $0.009^{*}$ & 9.521 & $1.799-50.402$ & $0.008^{*}$ \\
\hline ADT exposure duration (days) & Continuous & 0.169 & & & & 0.386 & & & \\
\hline
\end{tabular}

$H R$ hazard ratio, $C l$ confidence interval, PSA prostate-specific antigen, $A D T$ androgen deprivation therapy, $P S A T_{1 / 2}$ prostate-specific antigen half-life * statistically significant at $p<0.05$ 
significance $(p=0.094)$ when other factors were considered. Ultra-rapid response did not affect the risk significantly in the multivariate model $(p=0.238)$ as well as in the univariate analysis. Among factors other than $\mathrm{PSAT}_{1 / 2}$, Gleason score $>7$ (HR 1.472, 95\% CI 1.018-2.128, $p=0.040)$ was also significantly associated with increased CRPC risk.

Protective effect of intermediate response could be also observed in the multivariate model for overall survival. In the univariate analysis, Gleason score $>7$, rapid response, intermediate response, $\mathrm{T} 3$ and $\mathrm{T} 4$ stage showed association with overall mortality ( $p=0.006,0.018,0.011,0.013$, and 0.009 , respectively). Age $(p=0.243)$ and the duration of exposure to $\operatorname{ADT}(p=0.386)$ was not associated with overall survival as well. In the multivariate model, intermediate response was associated with significanly decreased mortality risk (HR 0.138, 95\% CI 0.033-0.584, $p=0.007$ ), followed by rapid response (HR 0.295, 95\% CI 0.109-0.797, $p=0.016$ ). Ultra-rapid response and slow response did not show association with overall mortality $(p=0.099,0.177)$. In addition to Gleason score (HR 3.127, 95\% CI 1.361-7.187, $p=$ 0.007 ), high $\mathrm{T}$ stage increased the mortality risk (T3: HR 4.987, 95\% CI 1.187-20.946, $p=0.028$; T4: HR 9.521, 95\% CI 1.799-50.402, $p=0.008$ ) in our model.

\section{Kaplan-Meier analysis further subcategorized by Gleason score}

Patients were further categorized into low to intermediate Gleason score $(\leq 7)$ and high Gleason score $(>7)$ strata to analyze the influence of poor differentiation on CRPC rate in the $\mathrm{PSAT}_{1 / 2}$ groups (Fig. 2). When stratified according to Gleason score group, 5-year CRPC rate for non-responding patients was exceptionally higher (71.4\%) compared to patients in rapid and slow response groups $(32.6 \%, p=0.020 ; 29.0 \%, p=0.027$; respectively) for the low to intermediate Gleason score stratum. Although CRPC rate was lower in intermediate and ultrarapid response groups, the difference was not significant (26.7\%, $p=0.076 ; 47.6 \%, p=0.405$, respectively). In the high Gleason score stratum, there were no statistically significant differences between the non-response group and other groups $(p=0.437,0.174$, and 0.468 , for ultrarapid, rapid, and slow response group, respectively). The only exception to this was intermediate response group, in which CRPC rate was significantly lower than in nonresponse group ( $44.0 \%$ vs. $66.7 \%, p=0.047)$.

\section{Discussion}

In the current study, we have attempted to identify patients at risk of developing CRPC by quantifying the response to ADT before the operation. By classifying according to preoperative $\operatorname{PSAT}_{1 / 2}$, we could identify patients at lower risk of developing CRPC from patients at high risk. Of note, our results indicate that group of patients with longer $\operatorname{PSAT}_{1 / 2}$ (30 to 45 days) can actually have lower CRPC rate, and that shorter $\mathrm{PSAT}_{1 / 2}$ is not necessarily associated with low risk of developing CRPC. (a) GS $\leq 7$

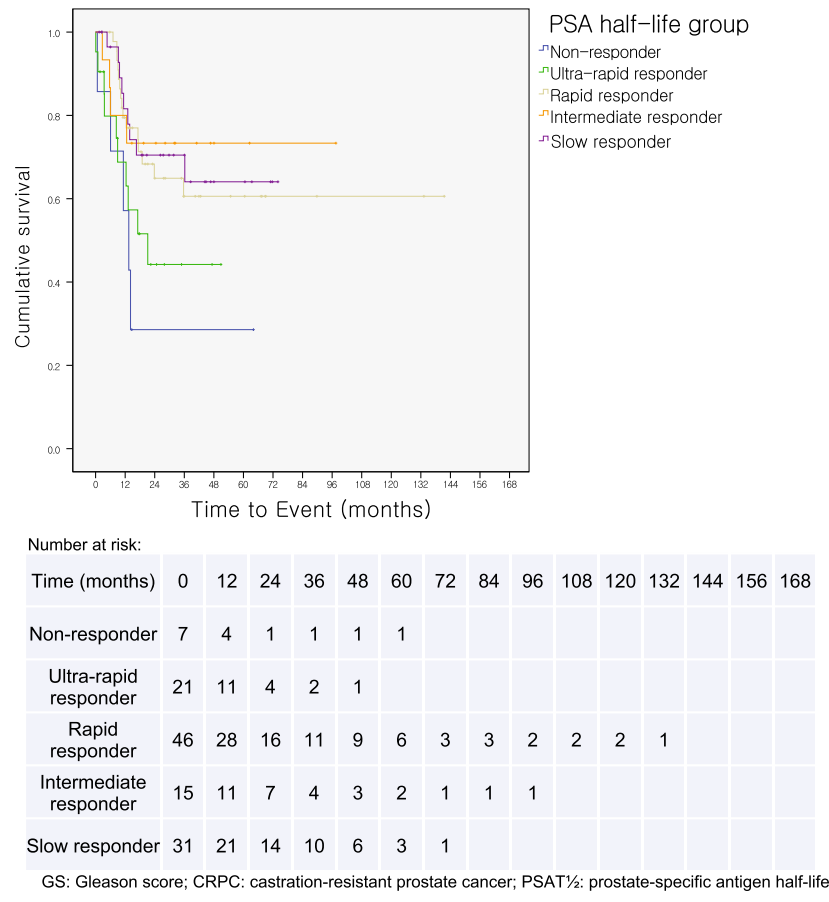

(b) $G S>7$

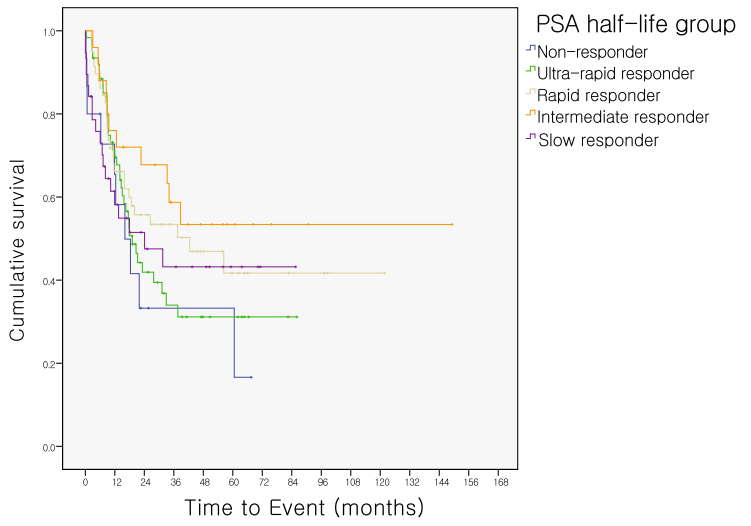

Number at risk:

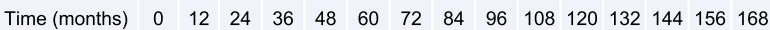

\begin{tabular}{l|l|l|l|l|l|l|} 
Non-responder & 15 & 8 & 3 & 2 & 2 & 2
\end{tabular}

Ultra-rapid

responder

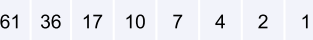

\begin{tabular}{ll|l|l|l|l|l|l|l|l|l|l|l} 
Rapid responder & 58 & 33 & 22 & 15 & 10 & 6 & 4 & 3 & 2 & 1 & 1
\end{tabular}

\begin{tabular}{ll|l|l|l|l|l|l|l|l|l|l|l|} 
Intermediate & 25 & 19 & 15 & 10 & 7 & 4 & 3 & 2 & 1 & 1 & 1 & 1
\end{tabular}

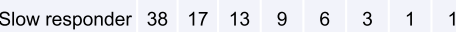

Fig. 2 Kaplan-Meier curve of PSA half-life groups for CRPC stratified by Gleason score. a Gleason $\leq 7$ stratum b Gleason $>7$ stratum 
By avoiding the need to wait for PSA to achieve nadir, PSAT $_{1 / 2}$ serves as a handy proxy for the response to ADT in the neoadjuvant setting. However, evidences so far are conflicting regarding whether the longer $\mathrm{PSAT}_{\frac{1}{2}}$ should be taken as a synonym for a longer remission period. In their study on $\mathrm{PSAT}_{1 / 2}$ during maximal androgen blockade in CRPC patients later treated with docetaxel, Lin et al. [7] concluded that $\mathrm{PSAT}_{1 / 2}>0.5$ months shortens CRPC-free survival and cancer-specific survival (HR $3.41, p=0.026)$. On the contrary, Kim et al. [10] suggested that $\operatorname{PSAT}_{1 / 2} \geq 0.5$ months after ADT is a condition associated with increased survival after docetaxel chemotherapy. Such contradictory results may in part come from the fact that $\mathrm{PSAT}_{1 / 2}$ over 0.5 months represents a different subset of patients in each study, as the cohort from the former study consisted of patients with median $\mathrm{PSAT}_{1 / 2}$ of 0.5 (IQR 0.1-34.2) months, while the median was 0.9 (IQR 0.5-2.0) months in the latter. Additionally, in the study by Kim et al. where mean values with standard deviations were also available, the mean was $3.5 \pm 9.2$ months, implying that the range included negative values. Because negative half-life corresponds to PSA doubling time [13], which means a failure to achieve nadir PSA and a resistance to ADT, it would be more appropriate to categorize the patients with negative values as a separate group.

In the current study, patients with $\mathrm{PSAT}_{1 / 2}$ shorter than 15 days presented with increased CRPC risk comparable to that of non-response group (Fig. 1a). A number of other studies also point out that a rapid response to ADT, measured by short $\operatorname{PSAT}_{1 / 2}[10,14]$ or time to nadir $[15,16]$, as an independent predictor of poor survival in patients with CRPC. The mechanism underlying the increase in CRPC risk for patients with steep PSA decline remains largely unknown, but postulated theories suggest that it is a result of the transcriptional effect of ADT on PSA production rather than prostate cancer cell death [16]; rapid removal of hormone-sensitive prostate cancer cells inducing an adequate environment for the growth of hormone-resistant prostate cancer cells [9]; or high probability of these subsets of patients containing cancers with low PSA production, such as cells with neuroendocrine differentiation [10] or cancer stem cells [17]. Westin et al. [18] demonstrated that the apoptotic activities are not uniformly increased in tumors responding to the androgen ablation with cellular atrophy and decreased cell proliferation rate. Considering that a decline in cell proliferation rate rapidly peaks within 7 days after castration but tumor apoptosis is found in only $30 \%$ of the patients [19], it is more likely that premature PSA decrease observed in our study was a result of halted proliferation rather than cell death. Acquisition of castration resistance in these dormant cells can result in regrowth despite the initial quick response to the androgen deprivation.
As expected, non-responders to ADT with negative PSAT $_{1 / 2}$ showed increased CRPC risk as demonstrated by the higher CRPC rate for the non-responding group (63.6\%) than the rapid response and slow response groups (Fig. 1a). These findings are in good agreement with previous literatures where an increase in PSA despite the initiation of ADT was associated with generally worse outcome. For instance, Matzkin et al. [5] showed that the median progression-free interval, for the patients whose lowest PSA level was more than $50 \mathrm{ng} / \mathrm{mL}$, was significantly shorter than those who achieved nadir (6 vs. 12 months, $p<0.0001$ ).

In our multivariate regression model, the Gleason score was the only parameter that was significantly associated with increased risk of CRPC (HR 1.472, 95\% CI $1.018-2.128, p=0.040$ ) other than $\operatorname{PSAT}_{1 / 2}$ (Table 2). An increase in Gleason score is known to be strongly associated with higher CRPC risk. It is shown in the study by Benaim et al. [20], that each unit of increase in Gleason score increases the risk of progression into CRPC by nearly $70 \%$ (HR $1.68,95 \%$ CI $1.3-2.1, \mathrm{p}<0.0001$ ). In the low to intermediate Gleason score $(\leq 7)$ patients, CRPC rate was significantly lower in the rapid and slow response groups when compared against the nonresponding group, while the gap was less explicit in the high Gleason score ( $>7)$ patients, as can be seen in Fig. 2. This increase of CRPC risk in poorly differentiated cancers is similar to the finding previously documented in the study by Benaim et al. [20] in which low and intermediate Gleason score patients experienced significantly longer remissions compared to Gleason score 8 to 10 patients ( 52.1 vs 25.2 months, $p=0.0006)$. A notable exception to this was the intermediate response group, which also showed significant association with markedly decreased risk of CRPC in the multivariate model (HR $0.397,95 \%$ CI $0.191-0.823, p=0.013)$. In the high Gleason score (>7) stratum, 5-year CRPC rate (44.0\%) remained lowest for the intermediate responders, especially compared to the non-response group $(60.0 \%, p=$ 0.047). It remains to be investigated in the future studies why the group with a $\mathrm{PSAT}_{1 / 2}$ of 30 to 45 days maintained relatively longer remission while CRPC rate in other groups uniformly increased to a level comparable to non-responders.

A possible explanation can be that this is because patients in the current study were exposed to neoadjuvant ADT before the normal prostate tissue was completely removed by surgery. Non-malignant human prostate tissue is reported to respond to ADT with apoptosis peaking 3-4 days after castration but returning to normal levels in approximately 2 weeks [21]. Ohlson et al. [19] proposed that this effect was due to the presence of remaining epithelium and basal cells. Considering that malignant cells show pronounced (7-fold) apoptotic 
activity starting from day 3-4 of castration regardless of Gleason score [19], latent proliferative activity could be seen as a reflection of a higher proportion of benign prostate cells being responsible for PSA secretion, thus slowing the decline in serum PSA level weeks after ADT.

The current study has some limitation to note. While the majority of patients (74.8\%) received adjuvant ADT consisting of 3-month interval LHRH agonist (Goserelin, Leuprolide and Triptorelin), a subset of patients went under combined androgen blockade with Bicalutamide (19.9\%), or even Bicalutamide monotherapy (5.4\%). Heterogeneous use of regimens may have interfered with maintained suppression of androgen receptor, and thus resulted in variations for PSA output. Although all patients went under routine biopsy process, its impact on PSA level cannot be determined in this study. As it is expected to cause certain level of prostatitis, raising serum PSA level, future study should consider incorporating this factor in the model. Since there is no data available regarding the serum testosterone levels of the cohort in the current study, we could not pinpoint the mechanism responsible for the observed risk increase in individual groups. Proper evaluation is necessary for the association between $\mathrm{PSAT}_{1 / 2}$ and the androgen activity to determine which mechanism is impaired specifically in nonresponders and ultra-rapid responders.

\section{Conclusion}

Patients with $\mathrm{PSAT}_{1 / 2}$ of 30 to 45 days after neoadjuvant therapy exhibited the lowest tendency to develop CRPC compared to patients with no response or ultra-rapid response who suffered high rate of adjuvant androgen deprivation therapy failure. A 1- to 3-month neoadjuvant ADT trial seems to be an effective way to identify patients who wound respond better to adjuvant ADT later, and prevent unnecessary long term ADT for patients who would not respond well to androgen deprivation.

\section{Additional files}

Additional file 1: Raw cohort data. Raw patient parameters used in the analysis (XLSX $68 \mathrm{~kb}$ )

\section{Abbreviations}

ADT: Androgen deprivation therapy; BCR: Biochemical recurrence; Cl: Confidence interval; CRPC: Castration-resistant prostate cancer; HR: Hazard ratio; IQR: Interquartile range; LHRH: Luteinizing hormone releasing hormone; PSA: Prostate-specific antigen; PSAT12/2: Prostate-specific antigen half-life

\section{Acknowledgements}

Not applicable.

\section{Funding}

There was no funding provided in the current research.
Availability of data and materials

All data generated or analysed during this study are included in this published article [and its Additional file 1].

\section{Authors' contributions}

YJK contributed in conception of the study, interpretation of the patient data and writing of the manuscript. WSJ, JKK, CYY, JYL, and WSH performed the statistical analyses of collected data. YJK and YDC contributed in conception and interpretation of the results. WSJ, JKK, CYY, JYL, WSH, and YDC were involved in the revision of essential parts of the manuscript. All authors read and approved the final manuscript. All authors have agreed to be accountable for all aspects of the work in ensuring that questions related to the accuracy or integrity of any part of the work are appropriately investigated and resolved.

\section{Ethics approval and consent to participate}

The current research was approved from the Severance hospital institutional review board (protocol number 4-2016-0506). Informed consent from the participants was waived by the institutional review board as the current study satisfied all of the following requirements for the waiver of informed consent:

-The research involved no more than minimal risk to the participants (retrospective data analysis of previously collected medical records).

-The waiver did not adversely affect the rights and welfare of the participants. -The research could not practicably be carried out without the waiver. -The participants were provided relevant information afterwards when necessary.

-The research was not subject to MFDS-FDA regulation.

\section{Consent for publication}

Not applicable.

\section{Competing interests}

The authors declare that they have no competing interests.

\section{Publisher's Note}

Springer Nature remains neutral with regard to jurisdictional claims in published maps and institutional affiliations.

Received: 22 January 2017 Accepted: 13 November 2017

Published online: 23 November 2017

\section{References}

1. Chandrasekar T, Yang JC, Gao AC, Evans CP. Mechanisms of resistance in castration-resistant prostate cancer (CRPC). Transl Androl Urol. 2015;4(3):365-80. doi:10.3978/j.issn.2223-4683.2015.05.02.

2. Hong JH, Kim IY. Nonmetastatic castration-resistant prostate cancer. Korean J Urol. 2014;55(3):153-60. doi:10.4111/kju.2014.55.3.153.

3. Elishmereni M, Kheifetz $Y$, Shukrun I, Bevan GH, Nandy D, McKenzie KM, et al. Predicting time to castration resistance in hormone sensitive prostate cancer by a personalization algorithm based on a mechanistic model integrating patient data. Prostate. 2016;76(1):48-57. doi:10.1002/pros.23099.

4. Fowler JE Jr, Whitmore WF Jr. Considerations for the use of testosterone with systemic chemotherapy in prostatic cancer. Cancer. 1982;49(7):1373-7.

5. Matzkin H, Eber $P$, Todd B, van der Zwaag R, Soloway MS. Prognostic significance of changes in prostate-specific markers after endocrine treatment of stage D2 prostatic cancer. Cancer. 1992;70(9):2302-9.

6. Arai Y, Yoshiki T, Yoshida O. Prognostic significance of prostate specific antigen in endocrine treatment for prostatic cancer. J Urol. 1990;144(6): 1415-9.

7. Lin GW, Yao XD, Zhang SL, Dai B, Ma CG, Zhang HL, et al. Prostate-specific antigen half-life: a new predictor of progression-free survival and overall survival in Chinese prostate cancer patients. Asian J Androl. 2009;11(4):443-50. doi:10.1038/aja.2008.36.

8. D'Amico AV, McLeod DG, Carroll PR, Cullen J, Chen MH. Time to an undetectable prostate-specific antigen (PSA) after androgen suppression therapy for postoperative or postradiation PSA recurrence and prostate cancer-specific mortality. Cancer. 2007;109(7):1290-5. doi:10.1002/cncr.22550.

9. Sasaki T, Onishi T, Hoshina A. Nadir PSA level and time to PSA nadir following primary androgen deprivation therapy are the early survival predictors for prostate cancer patients with bone metastasis. Prostate Cancer Prostatic Dis. 2011;14(3):248-52. doi:10.1038/pcan.2011.14. 
10. Kim M, Lee J, Jeong CW, Ku JH, Kim HH, Kwak C. Prostate-specific antigen kinetic profiles during androgen deprivation therapy as prognostic factors in castration-resistant prostate cancer. Urol Oncol. 2015;33(5):203 e1-9. doi: 10.1016/j.urolonc.2015.01.017.

11. Zilli T, Dal Pra A, Kountouri M, Miralbell R. Prognostic value of biochemical response to neoadjuvant androgen deprivation before external beam radiotherapy for prostate cancer: a systematic review of the literature. Cancer Treat Rev. 2016;46:35-41. doi:10.1016/j.ctrv.2016.03.016.

12. Armstrong AJ, Eisenberger MA, Halabi S, Oudard S, Nanus DM, Petrylak DP et al. Biomarkers in the management and treatment of men with metastatic castration-resistant prostate cancer. Eur Urol. 2012;61(3):549-59. doi:10.1016/ j.eururo.2011.11.009.

13. Banu E, Banu A, Medioni J, Levy E, Thiounn N, Mejean A, et al. Serum PSA half-life as a predictor of survival for hormone-refractory prostate cancer patients: modelization using a standardized set of response criteria. Prostate. 2007;67(14):1543-9. doi:10.1002/pros.20627.

14. Park YH, Hwang IS, Jeong CW, Kim HH, Lee SE, Kwak C. Prostate specific antigen half-time and prostate specific antigen doubling time as predictors of response to androgen deprivation therapy for metastatic prostate cancer. J Urol. 2009;181(6):2520-2524; discussion 5. doi:10.1016/j.juro.2009.01.104.

15. Tomioka A, Tanaka N, Yoshikawa M, Miyake M, Anai S, Chihara Y, et al. Nadir PSA level and time to nadir PSA are prognostic factors in patients with metastatic prostate cancer. BMC Urol. 2014;14:33. doi:10.1186/1471-2490-14-33.

16. Choueiri TK, Xie W, D'Amico AV, Ross RW, Hu JC, Pomerantz M, et al. Time to prostate-specific antigen nadir independently predicts overall survival in patients who have metastatic hormone-sensitive prostate cancer treated with androgen-deprivation therapy. Cancer. 2009;115(5):981-7. doi:10.1002/ cncr.24064.

17. Kyprianou N, Martikainen P, Davis L, English HF, Isaacs JT. Programmed cell death as a new target for prostatic cancer therapy. Cancer Surv. 1991;11:265-77.

18. Westin $P$, Stattin $P$, Damber JE, Bergh A. Castration therapy rapidly induces apoptosis in a minority and decreases cell proliferation in a majority of human prostatic tumors. Am J Pathol. 1995;146(6):1368-75.

19. Ohlson N, Wikstrom P, Stattin P, Bergh A. Cell proliferation and apoptosis in prostate tumors and adjacent non-malignant prostate tissue in patients at different time-points after castration treatment. Prostate. 2005;62(4):307-15. doi:10.1002/pros.20139.

20. Benaim EA, Pace CM, Roehrborn CG. Gleason score predicts androgen independent progression after androgen deprivation therapy. Eur Urol. 2002;42(1):12-7.

21. Staack A, Kassis AP, Olshen A, Wang Y, Wu D, Carroll PR, et al. Quantitation of apoptotic activity following castration in human prostatic tissue in vivo. Prostate. 2003:54(3):212-9. doi:10.1002/pros.10179.

\section{Submit your next manuscript to BioMed Central and we will help you at every step:}

- We accept pre-submission inquiries

- Our selector tool helps you to find the most relevant journal

- We provide round the clock customer support

- Convenient online submission

- Thorough peer review

- Inclusion in PubMed and all major indexing services

- Maximum visibility for your research

Submit your manuscript at www.biomedcentral.com/submit

) Biomed Central 